\title{
Primary microcystic/reticular schwannoma of the frontal bone: illustrative case
}

\author{
Xuemiao Zhao, ${ }^{1}$ Xinmu Zhou, MD, PhD, ${ }^{2}$ Xiao Chen, MD, ${ }^{1}$ Junqiao Pan, MD, ${ }^{1}$ and Bingrong Li, MD, PhD ${ }^{1}$ \\ Departments of ${ }^{1}$ Radiology and ${ }^{2}$ Pathology, The Fifth Affiliated Hospital of Wenzhou Medical University, Lishui City, Zhejiang Province, China
}

BACKGROUND To the best of the authors' knowledge, this is the first case of microcystic/reticular schwannoma arising in the frontal bone.

OBSERVATIONS An 18-year-old man presented to the authors' orthopedic clinic with a complaint of a progressively enlarging, painless mass in the frontal bone. It showed significant hyperintensity on T2-weighted imaging with progressive enhancement. Computed tomography combined with threedimensional reconstruction showed expansive bone destruction with a soft tissue mass in the left side of the frontal bone, without calcification inside or a sclerotic margin around it. The mass was a microcystic/reticular schwannoma as confirmed by surgical pathology.

LESSONS The authors report a rare case of a microcystic/reticular schwannoma arising in the frontal bone, with relatively comprehensive imaging data that enabled them to learn more about this tumor.

https://thejns.org/doi/abs/10.3171/CASE21175

KEYWORDS schwannoma; tomography; computer tomography; magnetic resonance imaging; case report

A rare type of schwannoma, microcystic/reticular schwannoma was first reported in $2008 .^{1}$ In the present case, we had comprehensive clinical and multiple imaging data, which led us to learn more about microcystic/reticular schwannoma.

\section{Illustrative Case}

\section{History}

An 18-year-old man who was a resident of Lishui City, a state in southeastern China, presented to our orthopedic outpatient clinic for the first time in January 2020 with a history of a progressively enlarging, painless mass in the left frontal bone that had first been discovered by accident 1 year earlier. On palpation, a painless and poorly mobile mass, about $2 \mathrm{~cm}$ in length, was found in the left forehead, with no skin ulceration. The patient underwent his first contrastenhanced magnetic resonance imaging (MRI) study the next day, and the mass was reported as an eosinophilic granuloma. However, the patient came to the hospital again in July because of further enlargement of the lesion. On the day of his presentation, physical examination and computed tomography (CT) showed that the left forehead mass was about 4 to $5 \mathrm{~cm}$ in length. Finally, the mass was resected approximately a week later and confirmed as a microcystic/reticular schwannoma.

Laboratory tests, including 7 tumor markers ( $\alpha$-fetoprotein, carcinoembryonic antigen, prostate-specific antigen, carbohydrate antigen [CA] 19-9, CA50, CA242, neuron-specific enolase), blood and urine routine tests, liver and kidney function tests, hepatitis virus tests, and Treponema pallidum and human immunodeficiency virus detection, revealed no positive findings.

\section{Imaging Findings}

Contrast-enhanced MRI performed in January 2020 showed a clean boundary mass in the left frontal bone, measuring about $2.7 \times 3.4 \times 2.8 \mathrm{~cm}$ in size. The lesion showed relative hypointensity on T1-weighted imaging (T1WI) compared with white matter (Fig. 1A), significant hyperintensity on T2-weighted imaging (T2WI) (Fig. 1B), relative hyperintensity on fluid-attenuated inversion recovery imaging (Fig. 1C), and a high apparent diffusion coefficient value (Fig. 1D). In contrast-enhanced sequences, the lesion showed mild heterogeneous enhancement on the axial images (Fig. 2A) and progressive enhancement on combined sagittal and coronal images (Fig. 2B and C).

ABBREVIATIONS CA = carbohydrate antigen; $\mathrm{CT}=$ computed tomography; $\mathrm{MRI}=$ magnetic resonance imaging; $\mathrm{T} 1 \mathrm{WI}=\mathrm{T} 1$-weighted imaging; $\mathrm{T} 2 \mathrm{WI}=\mathrm{T} 2$-weighted imaging INCLUDE WHEN CITING Published June 21, 2021; DOI: 10.3171/CASE21175.

SUBMITTED March 23, 2021. ACCEPTED April 2, 2021.

(c) 2021 The authors, CC BY-NC-ND 4.0 (http://creativecommons.org/licenses/by-nc-nd/4.0/). 

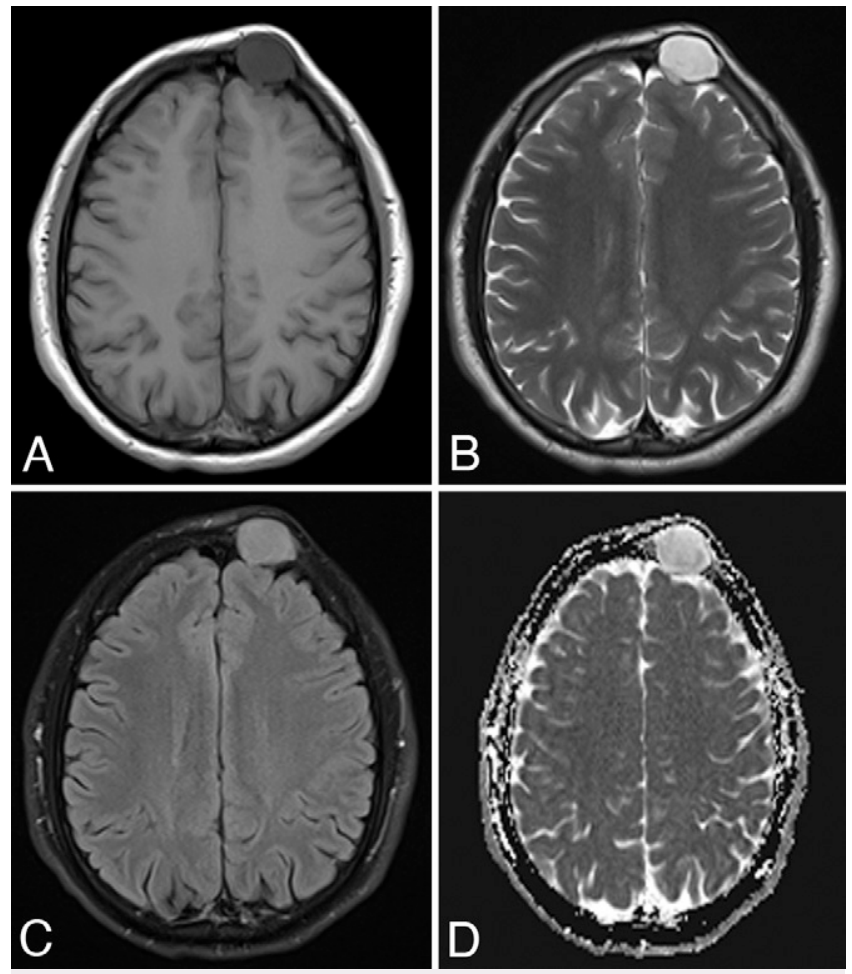

FIG. 1. A clean boundary mass in the left frontal bone, measuring about $2.7 \times 3.4 \times 2.8 \mathrm{~cm}$ in size, showed hypointensity on T1WI (A) and hyperintensity on T2WI (B) and fluid-attenuated inversion recovery $(\mathbf{C})$, which indicated that the lesion contained abundant water, while the high apparent diffusion coefficient value indicated unlimited diffusion (D).

CT performed in July 2020 demonstrated that bone destruction was present in the left frontal bone, without a sclerosing edge or obvious calcification (Fig. 3D), nearing which the cortex was incomplete (Fig. 3A).

\section{Operation and Pathology}

The operation revealed a soft frontal bone mass closely adherent to the endocranium (Fig. 4). Grossly, the lesion was incompletely encapsulated and locally invaded adjacent bone cortex, the cut surface of which was grayish yellow and jelly-like, measuring about $4.0 \times 4.0 \times 2.5 \mathrm{~cm}$ in size (Fig. 5A). Microscopic examination showed the spindle-shaped tumor cells had no obvious atypia and rare mitosis, arranging in a reticular, microcapsule-like structure within obvious mucinous degenerative stroma (Fig. 5B).

Immunohistochemistry revealed the following: vimentin,$+ \mathrm{S} 100+$, SOX-10+, CR+, glial fibrillary acidic protein negative, CD117-, CD34-, ETS-related gene positive, CD31+, podoplanin negative, desmin negative, smooth muscle actin positive (locally positive), AE1/ AE3-, epithelial membrane antigen negative, and Ki-67+ (about 5\%) (Fig. 5C). The final diagnosis was microcystic/reticular schwannoma.

\section{Discussion}

\section{Observations}

MRI showed that the lesion was water rich, without diffusion limitation on diffusion-weighted imaging, and accompanied by progressive
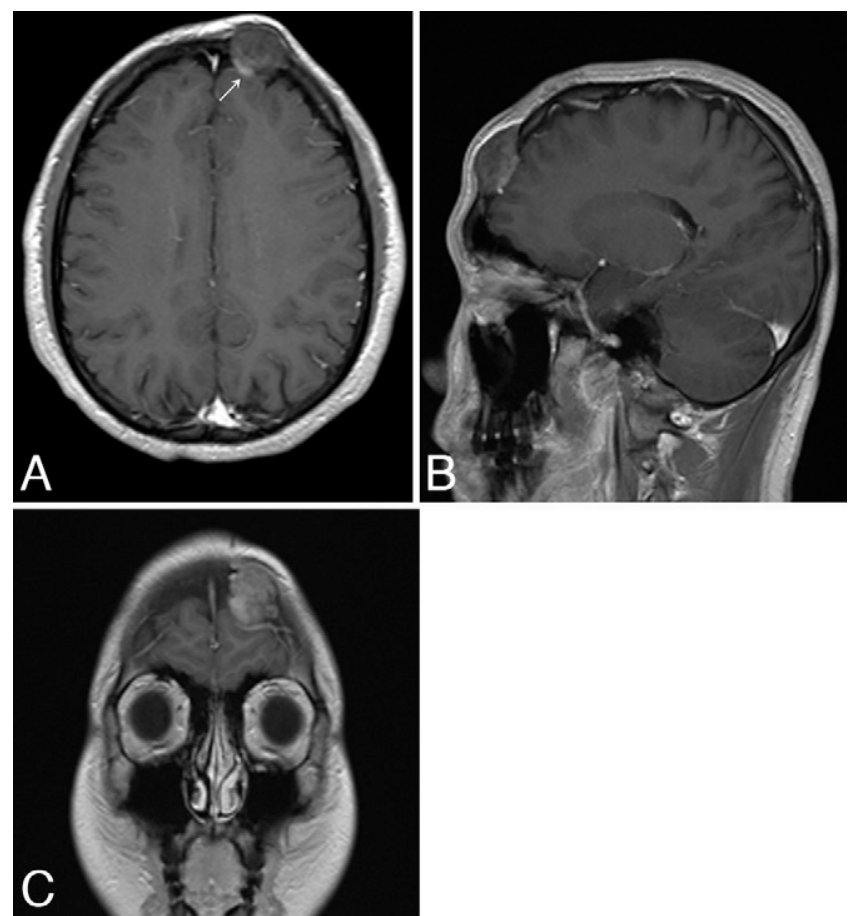

FIG. 2. The lesion showed heterogeneous mild to moderate enhancement with small flakes of relatively significant enhancement (arrow in A) on the axial image, sagittal image (B), and a large area of obvious enhancement on the coronal image $(\mathbf{C})$.

filling enhancement. CT showed that the lesion had an irregular border but no sclerotic edge, and the corresponding cranial plate became thin, even discontinuous. Combined CT and MRI revealed that the lesion enlarged significantly in 6 months. All of these observations
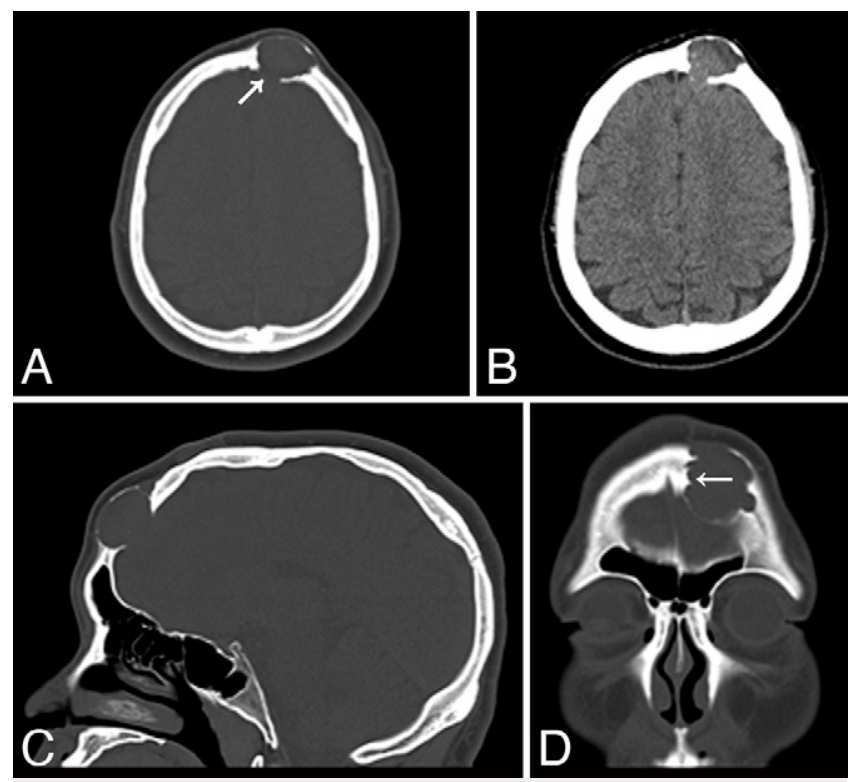

FIG. 3. The local bone cortex was intermupted incompletely (arrow in A), the edge was irregular (arrow in D), and there was no sclerotic edge or calcification in the lesion throughout axial (A and B), sagittal (C), and coronal (D) images. 


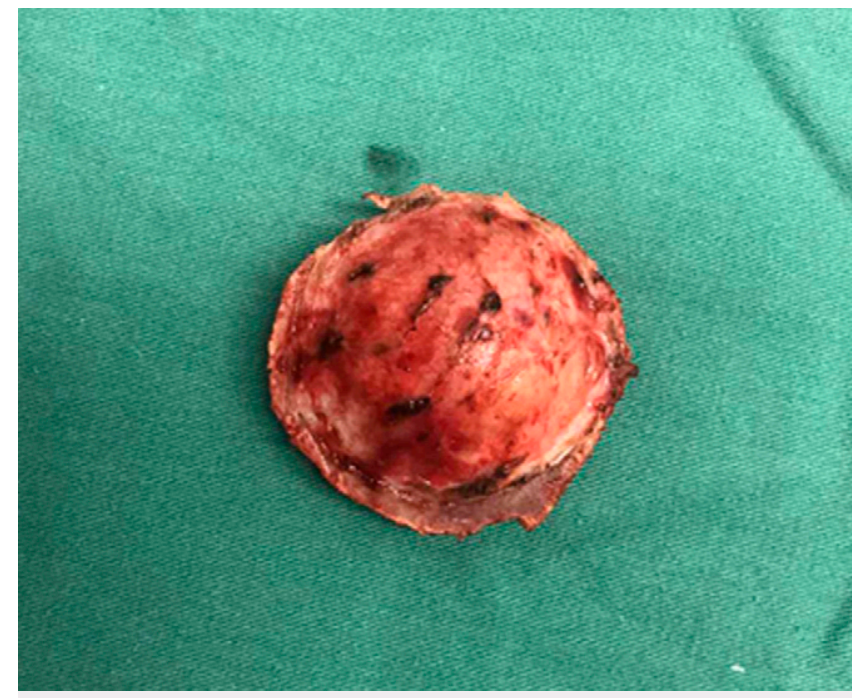

FIG. 4. General specimen of the operation. The margin was skull, and the center was tumor.

were sufficiently important that they contributed to a preoperative diagnosis. Microcystic/reticular neurinoma is rare; 36 cases had been reported globally as of January 2021 (only 28 cases in 17 articles in PubMed). It is extremely rare in bone (2 cases in total). ${ }^{2-10}$ To the best of our knowledge, the imaging findings described in this article have not been reported before.

\section{Lessons}

Before surgery was performed, the radiologists raised several possibilities. First, the lesion was deemed to be a water-/blood-rich disease according to its hyperintensity on T2WI, so that eosinophilic granuloma, lamellar meningioma, and lymphoma were excluded because their signal is similar to that of soft tissue. ${ }^{11,12}$ Second, although chondrogenic tumors, particularly those with mucus, might show significant hyperintensity on T2WI, they were hardly seen in the skull. Moreover, no calcification was seen on CT, which was another reason that a cartilage origin was unsupported. Regarding angiogenic tumors, the "gradually filling" enhancement was ample evidence (because the enhancement area of the lesion was enlarged throughout the axial, sagittal, and coronal images, among which there was a certain time interval; this was a reasonable speculation based on insufficient images and also an experience of imaging analysis). Nonetheless, this diagnosis was finally excluded because the enhancement in the arterial phase was far from a sufficient distinction. Unfortunately, we failed to make a definitive diagnosis. As a conclusion, our final inclination was a gelatinous or mucinous malignancy.

Most microcapsule/reticular schwannomas have an infiltrating growth pattern; therefore, resection is necessary. Differing from other malignant schwannomas, it had a favorable prognosis, and there has been no report of recurrence or metastasis. ${ }^{1}$

\section{Acknowledgments}

We extend thanks to Prof. Enfen Shu and Ming Luo for suggestions and help.
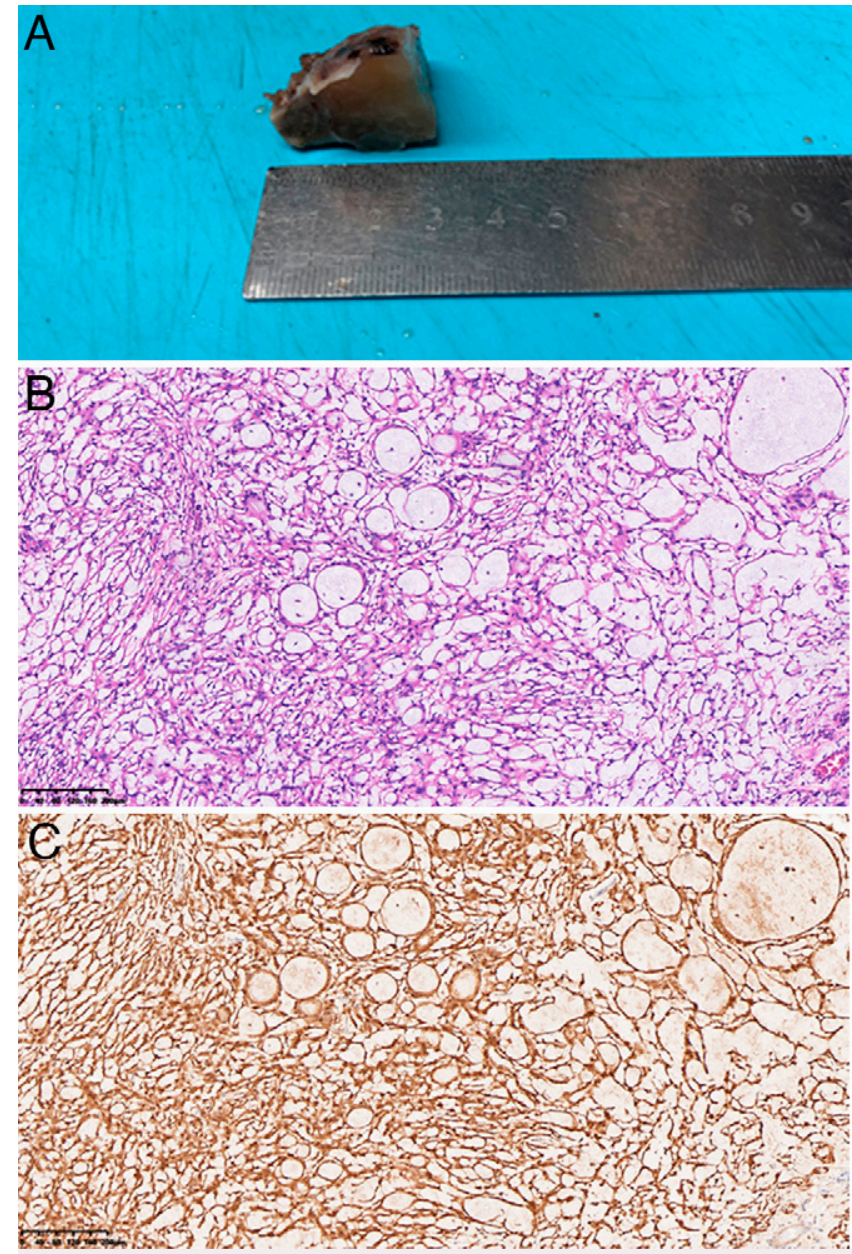

FIG. 5. A: The section of the specimen (one-quarter) showed that the mass was grayish-yellow and jelly like. B: The tumor cells were arranged in a reticular, microcapsule-like structure with mucinous stroma. Hematoxylin and eosin stain, original magnification $\times 100$. C: $\mathrm{S} 100$ staining was strongly positive. S100 stain, original magnification $\times 100$.

\section{References}

1. Liegl B, Bennett MW, Fletcher CDM. Microcystic/reticular schwannoma: a distinct variant with predilection for visceral locations. Am J Surg Pathol. 2008;32(7):1080-1087.

2. Tang SX, Sun YH, Zhou XR, Wang J. Bowel mesentery (meso-appendix) microcystic/reticular schwannoma: case report and literature review. World J Gastroenterol. 2014;20(5): 1371-1376.

3. Trivedi A, Ligato S. Microcystic/reticular schwannoma of the proximal sigmoid colon: case report with review of literature. Arch Pathol Lab Med. 2013;137(2):284-288.

4. Kienemund J, Liegl B, Siebert F, et al. Microcystic reticular schwannoma of the colon. Endoscopy. 2010;42(suppl 2):E247.

5. Gu MJ, Choi JH. Microcystic/reticular schwannoma of the esophagus: the first case report and a diagnostic pitfall. BMC Gastroenterol. 2014;14(1):193.

6. Liegl B, Bodo K, Martin D, et al. Microcystic/reticular schwannoma of the pancreas: a potential diagnostic pitfall. Pathol Int. 2011; 61(2):88-92. 
7. Bedir R, Semerci O, Fõndõk Güvendi G. Incidental cutaneous microcystic/reticular schwannoma in pilonidal sinus. Balkan Med J. 2019;37(1):52-53

8. Georgescu TA, Dumitru AV, Oproiu AM, et al. Cutaneous microcysticl reticular schwannoma: case report and literature review of an exceedingly rare entity with an unusual presentation. Rom J Morphol Embryol. 2018:59(1):303-309.

9. Yin Y, Wang T, Cai YP, et al. Microcystic/reticular schwannoma of the mandible first case report and review of the literature. Medicine (Baltimore). 2015:94(45):e1974.

10. Liu C, Yan L, Liu Q, et al. Lumbar intraspinal microcystic/reticular schwannoma: case report and literature review. Medicine (Baltimore). 2018;97(39):e12474.

11. Guo R, Xu P, Cheng S, et al. Comparison of nasopharyngeal MR, ${ }^{18}$ F-FDG PET/CT, and ${ }^{18}$ F-FDG PET/MR for local detection of natural killer/T-cell lymphoma, nasal type. Front Oncol. 2020;10:576409.
12. Woertler K. Benign bone tumors and tumor-like lesions: value of cross-sectional imaging. Eur Radiol. 2003;13(8):1820-1835.

\section{Disclosures}

The authors report no conflict of interest concerning the materials or methods used in this study or the findings specified in this paper.

\section{Author Contributions}

Acquisition of data: Zhou, Chen, Pan. Analysis and interpretation of data: Zhou, Pan. Drafting the article: Li, Zhao. Critically revising the article:

Zhao. Reviewed submitted version of manuscript: Li, Zhao. Approved the final version of the manuscript on behalf of all authors: Li.

\section{Correspondence}

Bingrong Li: The Fifth Affiliated Hospital of Wenzhou Medical University, Lishui City, Zhejiang Province, China.1528685422@qq.com. 